\title{
Fluid movement across synovium in healthy joints: role of synovial fluid macromolecules
}

\author{
J R Levick, J N McDonald
}

Synovial fluid is not a static pool, but is continually being absorbed and replenished by the synovial lining of the joint cavity (synovium, synovial intima), which is approximately $20 \mu \mathrm{m}$ thick (rabbit knee) to approximately $60 \mu \mathrm{m}$ thick (human knee). The three key elements for fluid turnover are the synovial capillary, synovial interstitium, and the lymphatic drainage system.

Synovial capillaries. Normal areolar and adipose synovium have a row of capillaries $\sim 5 \mu \mathrm{m}$ (rabbit) to $\sim 30 \mu \mathrm{m}$ (man) beneath the surface, many bearing fenestrations (membranes of high permeability to water), often on the side facing the joint cavity. ${ }^{1-3}$ The high capillary density, superficial location, and fenestral orientation are well adapted for synovial fluid formation and nutrient supply. Synovial fluid is formed primarily by ultrafiltration of plasma across the fenestral membranes, driven by a net imbalance in the 'Starling pressures' acting across the membrane. The Starling pressure imbalance is the pressure drop from capillary plasma to synovial interstitium, minus the difference in effective colloidal osmotic pressure (COP) across the capillary wall. Experimental studies of how capillary blood pressure, plasma COP and intra-articular pressure affect net trans-synovial flow have been reviewed elsewhere. ${ }^{45}$ The influence of intra-articular (IA) colloids, however, has only recently begun to be studied and is considered here. Although capillary ultrafiltrate is the raw material for synovial fluid formation, the synovial lining cells also actively secrete the glyosaminoglycan hyaluronan and the glycoprotein lubricin to produce the highly viscous, lubricating synovial fluid. ${ }^{6}$

Synovial interstitium. The path into the joint cavity from a capillary, and from joint cavity to a lymphatic vessel in the subsynovium, passes between the lining cells. These intercellular gaps, several $\mu \mathrm{m}$ wide, contain a complex fibrous matrix (type I, III, and V collagen fibrils, type VI collagen microfibrils, hyaluronan, chondroitin and heparan proteoglycans, possibly keratan sulphate, fibronectin) in open contact with IA fluid. The hydraulic conductivity of the matrix is about $10^{-11} \mathrm{~cm}^{4} \mathrm{~s}^{-1} \mathrm{dyn}^{-1}$ or less, ${ }^{78}$ which helps to reduce the rate of escape of IA fluid when joint pressure is increased (for example in flexion).

Effect of joint motion and angle. Intra-articular fluid pressure (IAP) is an important factor affecting net flow across synovial interstitium: it opposes capillary filtration by increasing pericapillary interstitial pressure, and it promotes drainage from joint cavity to subsynovium. IAP is affected by movement of a joint, linking joint motion to fluid transport. Active or passive flexion of a normal joint can increase IAP to just above atmospheric pressure (much greater for effusions), whereas in extension IAP is a few $\mathrm{cm} \mathrm{H}_{2} \mathrm{O}$ less than atmospheric. At subatmopheric pressures, net flow is often into the joint cavity, and at supra-atmospheric pressures net flow is directed out of the healthy cavity. ${ }^{45}$ Synovial lymphatic system. A plexus of terminal lymph vessels is found at the subsynoviumsynovium border and drains away fluid, macromolecules, and particles that have escaped from the joint cavity. ${ }^{6910}$ Subsynovium comprises loose areolar tissue (substantial areas), and fatty and fibrous tissue. Areolar subsynovium connects with surrounding connective tissue planes and, like areolar tissue elsewhere, acts as a compliant, low-pressure 'sink' when fluid is driven into it, as in experiments described here.

\section{Intra-articular albumin and trans- synovial flow}

Edlund established that IAP promotes the escape of saline from the synovial cavity, and that the pressure-outflow relation steepens at pathological IAPs because the hydraulic resistance of the synovial lining decreases. ${ }^{11}$ Normal synovial fluid, however, is more complex than saline: its most abundant macromolecule, albumin ( $40-45 \%$ of plasma concentration), endows the fluid with COP, while hyaluronan $(\sim 3 \mathrm{~g} / \mathrm{l})$ dominates the viscosity.

Albumin not only increases COP but also increases viscosity modestly. To study its effect on trans-synovial flow in our laboratory, we inserted two cannulae into the joint cavity of a rabbit knee under anaesthesia. One cannula recorded IAP and the other infused albumin solution from a gravity-driven reservoir, the vertical height of which regulated IAP. An intervening drop counter recorded the rate of absorption of infusate. Flows were measured in the steady state, and after a small correction for wall creep this was equated with net transsynovial flow, $\dot{Q}_{\text {s }}$ (net absorption rate). (The absorbate mostly passes into subsynovium, but transcapillary flow is involved also-see below.) The procedure was to infuse known albumin concentrations alternately with Krebs solution; after 15 minutes of infusion, the joint was flushed with the new infusate. No hyaluronan was present. $\dot{Q}_{s}$ in the presence of albumin was then expressed as a fraction of $\dot{Q}_{\text {s }}$ for Krebs solution; this will be referred to as the 'fractional flow'. 


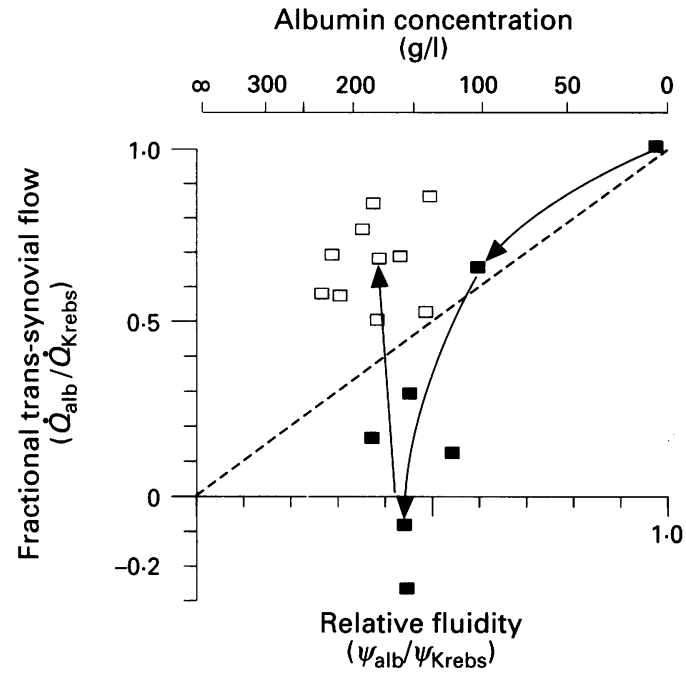

Figure 1 Effect of interstitial albumin on fractional transsynovial flow in vivo( $\square$ ) and after death ( $\square$ ) at 3-6 cm $\mathrm{H}_{2} \mathrm{O}$ intra-articular pressure, plotted as a function of solution fluidity, $\psi$. Fractional flow is net trans-synovial flow of albumin solution relative to that of Krebs solution: $Q_{a l b} / Q_{\text {Krebs. }}$ Relative intra-articular fluidity is $\psi_{\text {alb }} / \psi_{\text {Krebs. }}$. Arrowed lines unite a sequence of results in a single joint. Dashed line of equality represents a simple porous medium in which fractional change in flow equals change in relative fluidity of liquid.

The effect of albumin on fractional flow in vivo is shown by the filled squares in figure 1 . The $x$ axis here is 'relative fluidity'. Fluidity $(\psi)$, the reciprocal of viscosity $(\eta)$, was plotted because, according to Darcy's law of flow, flow $\dot{Q}$ through a rigid macroscopic porous medium (e.g. sand) is inversely proportional to viscosity:

$$
\dot{Q}=\kappa(A / \eta)(d P / d x)=\psi \kappa A(d P / d x)
$$

where $\kappa$ specific hydraulic conductivity, $A=$ bed area. When the flow of albumin solution is divided by that of the solvent (Krebs solution here), fractional flow should equal the

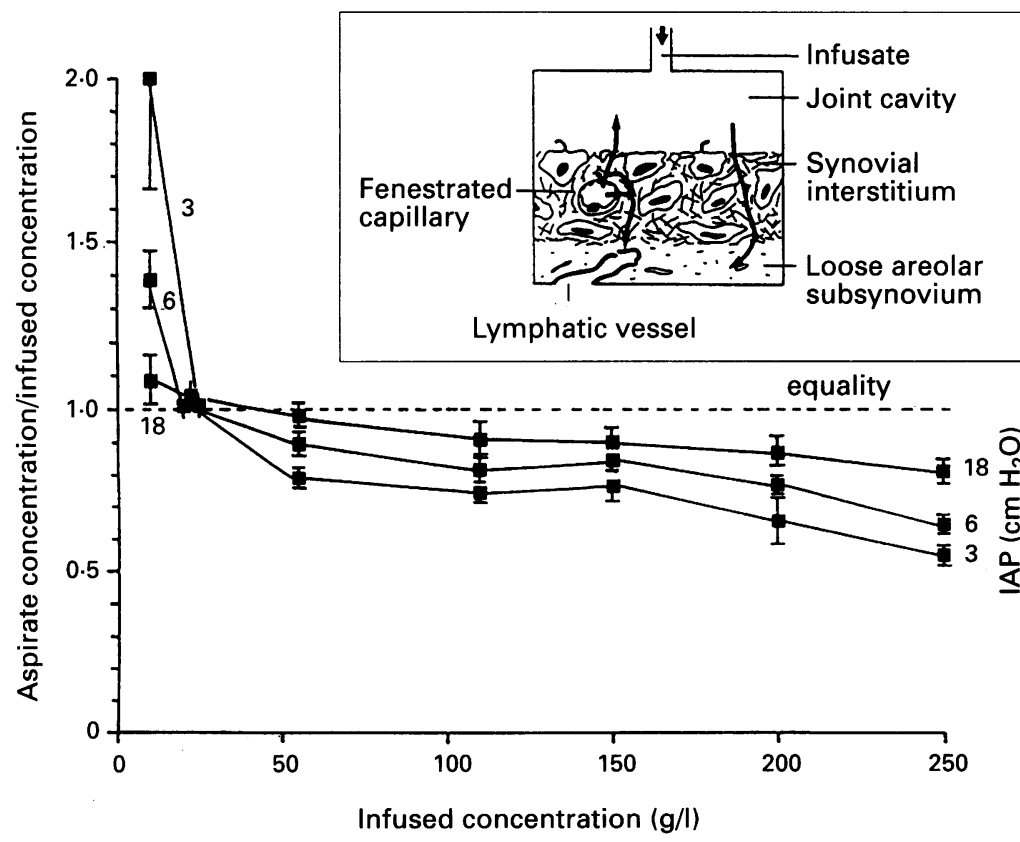

Figure 2 Albumin concentration in joint cavity of rabbit knee relative to infused concentration after continuous infusion for 15 minutes preceded by triple flush, plotted as a function of infused concentration (mean, SEM). ${ }^{24}$ Results at three different intra-articular fluid pressures (IAPS) shown. Inset shows proposed explanation, based on net transsynovial consisting of two oppositely directed flows. relative fluidity (reciprocal of relative viscosity) if Darcy's law applies and pressure gradient $d P / d x$ and bed area $A$ are constant. The dashed line of equality in figure 1 is thus a guide to whether net transynovial flow obeys Darcy's law. Net $\dot{Q}_{\text {s }}$ in vivo did not follow this line, however: at IAPs of 3-6 $\mathrm{cm} \mathrm{H}_{2} \mathrm{O}$, albumin reduced net $\dot{Q}_{\mathrm{s}}$ more than expected from the reduced fluidity. At very high, non-physiological concentrations, net flow even reversed direction to a net filtration into the cavity. The deviation below the Darcy line and eventual reversal is caused by capillary filtration into the cavity (see later), which is increased by the colloid osmotic pressure of the IA extravascular fluid (Starling's principle). Capillary filtration into the cavity partially or wholely offsets drainage from the cavity via intercellular spaces, reducing the net trans-synovial absorption rate. Intra-articular albumin thus demonstrably permeated the lining to reach the pericapillary spaces. The above interpretation was supported by postmortem experiments (see later), but before considering these, two other features in vivo are notable.

Are pericapillary COP and intra-articular COP equal? The effect of IA albumin on net $\dot{Q}_{\mathrm{s}}$ was less than expected from the effect of intravascular albumin on $\dot{Q}_{\mathrm{s}},{ }^{12}$ if one were to assume that pericapillary albumin concentration equals that in the joint cavity. This suggested the possibility that pericapillary albumin concentration might be less than in the joint cavity here, despite the proximity of the capillaries. This inference was supported by modelling studies (see below). It appears that synovial fluid protein concentration and COP are not necessarily true measures of the values acting at the capillary wall at high filtration rate. The difference may be small normally, however.

Inflow and outflow simultaneously across the synovial lining in vivo. In the above experiments, IA fluid was aspirated for analysis after the 15 minute infusion period. To our initial surprise, IA albumin concentration was less than that being infused, despite the prolonged infusion period and a preceding triple flush (fig 2). The aspirate/infusate concentration ratio decreased with increasing infusate concentration, and increased closer to unity with increasing IAP. This led us to suspect that an internal circulation of fluid was occurring (fig 2 inset). The COP of pericapillary albumin increases the rate of capillary ultrafiltration into the joint cavity by the Starling principle, leading to IA dilution. At the same time, outflow occurs through the interstitial pathway parallel to and some micrometres distant from the capillary. A net trans-synovial outflow (absorption) is recorded when interstitial outflow from the cavity exceeds capillary filtration rate into it. For this hypothesis to be tenable, however, IA albumin must diffuse sufficiently rapidly up the stream of capillary filtrate to maintain a filtration-enhancing COP in the pericapillary region. Is this a realistic possibility? To address this, a mathematical model of trans-synovial flow and albumin diffusion was constructed, as follows. 
Tissue
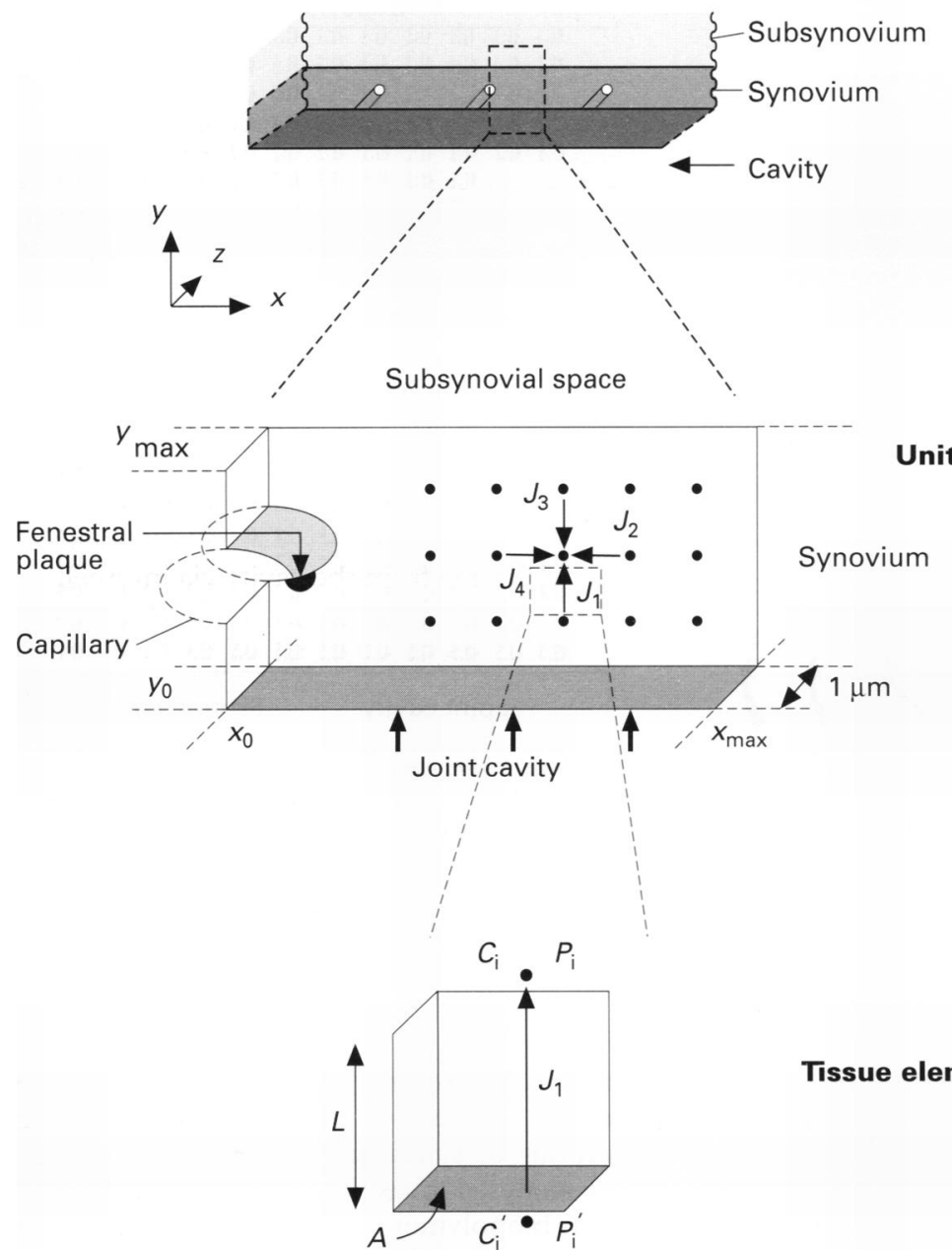

Figure 3 Two dimensional model for trans-synovial flow. ${ }^{8}$ The orientation is inverted compared with figure 2 in order to make the $\mathrm{y}$ axis positive. Synovial lining in situ (Tissue) is subdivided into identical unit cells (middle), and the unit cell further subdivided into very small blocks (Tissue element). $\mathrm{J}=$ flux of water or albumin in given direction; $\mathrm{P}_{\mathrm{i}}, \mathrm{C}_{\mathrm{i}}=$ interstitial pressure and protein concentration at defined point; $\mathrm{A}, \mathrm{L}=$ surface area and length of each tissue element.

\section{Modelling trans-synovial flow and protein transport}

The two dimensional model (fig 3) was based on mean morphometric values for rabbit knee synovium: ${ }^{8}$ anatomical heterogeneity is neglected. The core of the model is a basic repeating unit consisting of a half capillary with an orientated fenestral cluster and a neighbouring tissue domain. Quantities such as

Biophysical aspects of interstitial transport: the properties are governed primarily by the concentration of interstitial biopolymers, especially glycosaminoglycan, the volume fraction of which is $\theta$

\begin{tabular}{|c|c|c|}
\hline Property & Key biophysical relations ${ }^{\star}$ & Reference/assumption \\
\hline $\begin{array}{l}\text { Hydraulic conductance } \\
\text { Solute exclusion } \\
\text { Reflection coefficient } \\
\text { Effective viscosity } \\
\text { Water flow } \\
\text { Restricted diffusion } \\
\text { Albumin transport by } \\
\text { diffusion and convection }\end{array}$ & $\begin{array}{l}k_{\mathrm{i}}=\kappa_{\mathrm{i}} / \eta_{\mathrm{i}} L \quad \kappa=1.6 \times 10^{-18} \cdot \theta^{-2 \cdot 3} \\
K_{\mathrm{AV}}=\exp \left\{-\left(\theta / r_{\mathrm{f}}^{2}\right)\left(r_{\mathrm{f}}+r_{\mathrm{s}}\right)\right\} \quad \phi=K_{\mathrm{AV}} / \mathrm{e}^{-\theta} \\
\sigma_{\mathrm{i}}=(1-\phi)^{2} \\
\eta_{\mathrm{i}}=f\left(\eta_{\text {bulle }} \phi, r_{\mathrm{s}}\right) \\
\mathcal{f}_{\mathrm{v}}=k_{\mathrm{i}}\left\{\Delta P_{\mathrm{i}}-\sigma_{\mathrm{i}} \Delta \pi_{\mathrm{i}}\right\} \\
D_{\mathrm{res}}=D \cdot \exp \left\{-\left(\theta / r_{\mathrm{f}}^{2}\right)^{0.5}\left(r_{\mathrm{f}}+r_{\mathrm{s}}\right)\right\} \\
\mathcal{f}_{\mathrm{s}}=f\left(\Delta C_{\mathrm{i}}, \operatorname{Pec}\right) \\
\quad \text { where } \operatorname{Pec}=\mathcal{F}_{\mathrm{v}}\left(1-\sigma_{\mathrm{i}}\right) / M_{\mathrm{i}} A\end{array}$ & $\begin{array}{l}14 \\
1516 \\
1617 \\
\text { Particle in tube model } \\
19 \\
20 \\
\text { Non-linear transport } \\
\text { equation }\end{array}$ \\
\hline
\end{tabular}

${ }^{\star}$ Corrections for cell volume fraction, collagen fibril volume fraction, and tortuosity factors are not shown here, for simplicity. $A=$ area; $C=$ concentration; $D_{\text {res }}=$ restricted diffusion coefficien within matrix; $\mathfrak{f}=$ transport rate; $K_{\mathrm{AV}}=$ fraction of extrafibrillar water space available to solute; $k=$ hydraulic conductance of unit area of material; $L=$ length of path; $M=$ diffusional permeability; Pec $=$ Peclet number; $r=$ radius of solute or biopolymer chain; $\Delta P_{\mathrm{i}}=$ pressure difference across a small element of tissue; $\theta=$ volume fraction occupied by a component; $\phi=$ solute partition coefficient in extrafibrillar space; $\eta=$ viscosity; $\kappa=$ specific hydraulic conductivity of material; $\pi=$ colloid osmotic pressure due to plasma protein (oncotic pressure); $\sigma=$ osmotic reflection coefficient; $\mathrm{f}=$ fibre (molecular chain); $\mathrm{i}=$ interstitium; $\mathrm{s}=$ solute; $\mathrm{v}=$ volume capillary wall conductance and interstitial hydraulic resistance were evaluated from published data. The tissue was subdivided into tiny cubic elements and protein flux across each face of the element calculated by the nonlinear membrane transport equation of Patlack et $a l^{13}$ for combined diffusional and convective (wash-along) transport. Each element was treated, in effect, as a leaky porous membrane and was allotted a finite concentration difference, pressure drop, hydraulic resistance, partial albumin exclusion, albumin reflection coefficient, and restriction to diffusion. All these material properties were related to interstitial biopolymer concentration (glycosaminoglycan, etc) by random fibre matrix theory (table). ${ }^{13-20}$ Capillary hydraulic permeability was represented by discrete sites (patch of fenestrations): the simplifying assumption of uniform capillary permeability to water (widely used in mathematical models) causes significant errors. The numerical solutions led to several useful insights into transsynovial flow, as follows.

Theoretical support for simultaneous bidirectional flow across synovium. Figure 4 shows the solution for one particular set of experimental boundary conditions (IAP, COP, etc). Over the synovial surface as a whole, a net outflow from the cavity is predicted (absorption), due to flow through interstitium remote from the capillary;

but there is also a smaller, localised inflow through the interstitium immediately overlying the capillaries, caused by capillary ultrafiltration. There is thus a local circulation of extravascular fluid and this explains much of the IA dilution observed experimentally (fig 2 ). Similar bidirectional trans-synovial flow patterns are computed at lower IAP-COP combinations such as might occur in vivo. The experiments and model thus reveal a novel mechanism for synovial fluid turnover in a stationary joint, provided that the joint angle is such that IAP is greater than subsynovial pressure.

Pericapillary COP v intra-articular COP. Most of the hydraulic permeability of a synovial capillary is concentrated in a tiny section of its perimeter, namely the patch of fenestrations. Filtration velocity $(\mathrm{cm} / \mathrm{s})$ is therefore orders of magnitude greater than if the same flow $\left(\mathrm{cm}^{3} / \mathrm{s}\right)$ occurred uniformly across the entire area of wall $\left(\mathrm{cm}^{2}\right)$. High filtration velocities tend to wash interstitial plasma protein molecules away from the outside of the fenestrations, reducing their local concentrations (fig 4, right). Despite this, a certain perifenestral protein concentration is preserved by diffusion against the ultrafiltration stream along the locally steepened concentration gradient (rather as a dye can diffuse up a sluggish stream), but the resulting perifenestral concentration is always less than IA concentration to some degree. This helps explain why a given IA concentration of albumin had apparently less effect on fluid exchange than intracapillary albumin-a feature noted earlier.

In the above experiments, extravascular albumin was supplied via an infusion line. Can 


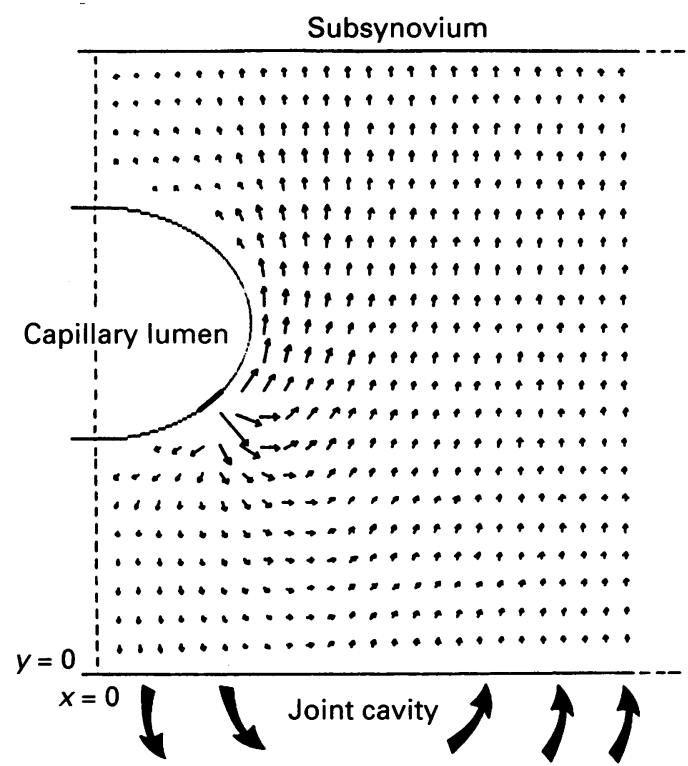

\section{Albumin field}

Subsynovium

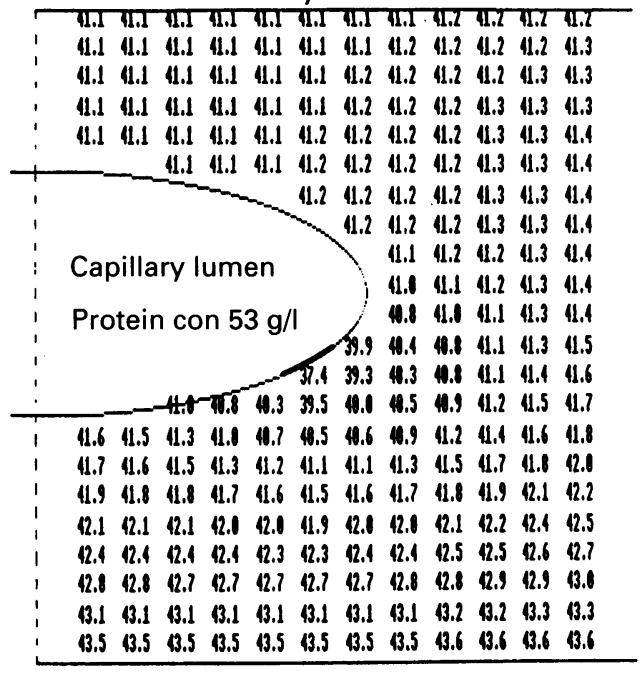

Joint cavity

Figure 4 Predicted bidirectional pattern of flow across synovial interface with joint cavity (left) and corresponding interstitial albumin concentrations (right: $x$ axis expanded for clarity). ${ }^{8}$ Dark bar on capillary wall shows site of patch of fenestrations. Boundary conditions: intra-articular pressure (IAP) $6 \mathrm{~cm} \mathrm{H} \mathrm{H}_{2} \mathrm{O}$; subsynovial pressure atmospheric; intraarticular albumin concentration $44 \mathrm{~g} /$; capillary pressure = plasma colloid osmotic pressure. Left: Thin arrows are flow vectors; flow into cavity $0.2 \mu \mathrm{l} / \mathrm{min}$; flow out of cavity $10.7 \mu \mathrm{l} / \mathrm{min}$; capillary filtration fraction 0.044 . Larger inflow/ outflow ratios occur upon increasing intra-articular albumin concentration or decreasing IAP. Right: Numbers represent albumin concentration in available, non-excluded interstitial water. Note the substantial dilution immediately around the filtering fenestral patch (thickened line). Over the capillary, albumin is diffusing out of the cavity against the inward filtration stream.

similar perifenestral gradients of plasma protein exist normally? To answer this, we must consider the normal route(s) of entry of plasma protein into the synovial interstitium and synovial fluid. The fenestral membrane itself has a high protein reflection coefficient $\left(0.8\right.$ for albumin $\left.{ }^{3}\right)$ and it is generally accepted that much of the extravascular plasma protein mass, especially for the larger molecular species, enters interstitium via the capillary large pore system, which comprises the vesicular transport system, continuous large pores or both. ${ }^{21}$ Thus much protein enters interstitium at regions spatially separate from the site of fenestral filtration, and perifenestral gradients analogous to those in figure 4 may therefore occur normally. As noted above, this would imply that intra-articular COP is only an approximation to the COP around the capillary fenestrations. The goodness of this approximation varies with filtration rate.

The model predictions for IA dilution and fractional flow ${ }^{8}$ broadly fitted the scattered results of figures 1 and 2 . The variability of $\dot{Q}_{s}$ in rabbit joints is a consistent feature in our experience.

\section{Relation between synovial lining permeability and biopolymer concentration}

The net concentration of biopolymers (glycosaminoglycans, proteoglycans and glycoproteins) in synovial interstitium is a key factor governing hydraulic resistance and permeability to macromolecules. For the model, a biopolymer concentration of $\sim 13.9 \mathrm{mg} / \mathrm{ml}$ of extrafibrillar space (interstitial space excluding collagen fibrils) was deduced from experi- mental estimates of synovial hydraulic resistance. ${ }^{822}$ Recently, the very small (mg) quantities of synovium in a rabbit knee have been analysed directly and quantitatively for certain biopolymers. The net concentration of chondroitin sulphate, heparan sulphate, and hyaluronan is $\sim 3.9 \mathrm{mg} / \mathrm{ml}$ of extrafibrillar space $^{23}$ (recalculated). This is substantially less than the theoretical biopolymer content of $\sim 13.9 \mathrm{mg} / \mathrm{ml}$. The difference is partly explained by the existence of unassayed biopolymers (for example glycoproteins, proteoglycan core-present on immunohistochemistry); indeed, similar discrepancies occur in other tissues if such components are neglected. ${ }^{22}$ The attempt to match biochemical composition and physiological properties in synovium is thus incomplete at present.

\section{Trans-synovial flow in absence of} capillary filtration: a viscosity anomaly Killing an animal abolishes capillary filtration, and this provided direct evidence that the deviation of fractional flow below the Darcy line in vivo (filled squares in figure 1) was caused by capillary filtration. When the animal was killed the low net outflows induced by albumin increased to much greater values (open squares in figure 1). Thus depression of trans-synovial outflow below the Darcy line in vivo is caused by microvascular filtration.

Trans-synovial flows of albumin solution post mortem lay above the Darcy line, rather than along it as one might expect upon first consideration. Experimental design may have exaggerated the deviation, ${ }^{24}$ but there is a fundamental theoretical reason why the fluidity of interstitial fluid should be different from that 
of the IA solution. The reason is that interstitial biopolymers exclude albumin from a fraction of the water space. ${ }^{25}{ }^{26}$ Around any biopolymer chain there is an annular space into which the centre of mass of a large globular solute cannot penetrate, because of the solute's finite radius (steric exclusion), but into which the much smaller water molecule has free access. The greater the glycosaminoglycan concentration, the greater is this excluded volume fraction ${ }^{15} 25$ (table). Since the trans-synovial pressure gradient must drive some water and electrolytes through the albumin-excluded space, in addition to driving albumin solution through the albumin-available space, the effective relative fluidity is a flow-weighted average between that of the saline solution (relative fluidity 1.0 ) and the albumin solution in the non-excluded space (fluidity $<1 \cdot 0$ ). Thus the effective interstitial fluidity is greater (closer to $1 \cdot 0$ ) than that of the feeding phase, as seen post mortem (fig 1). In other words, steric exclusion of interstitial plasma proteins reduces the effective viscosity of the permeating fluid. This effect was obscured in vivo by the large, COPinduced changes in capillary filtration, except perhaps at low albumin concentrations.

Anomalous viscosity in narrow channels A first-approximation treatment of anomalous viscosity was developed by analogy with laminar flow of particulate suspensions (such as blood) down narrow cylindrical tubes; here, steric exclusion at the walls produces a similar anomalous low viscosity (Fahreus-Lindquist effect ${ }^{27}$ ). Flow through the spaces between cylindrical fibres exhibits Poiseuille-like laminar patterns, ${ }^{28}$ because the channel is in effect an 'inside-out' tube. For a narrow cylindrical tube (radius $R_{\text {tube }}$ ) with laminar flow, Whitmore ${ }^{18}$

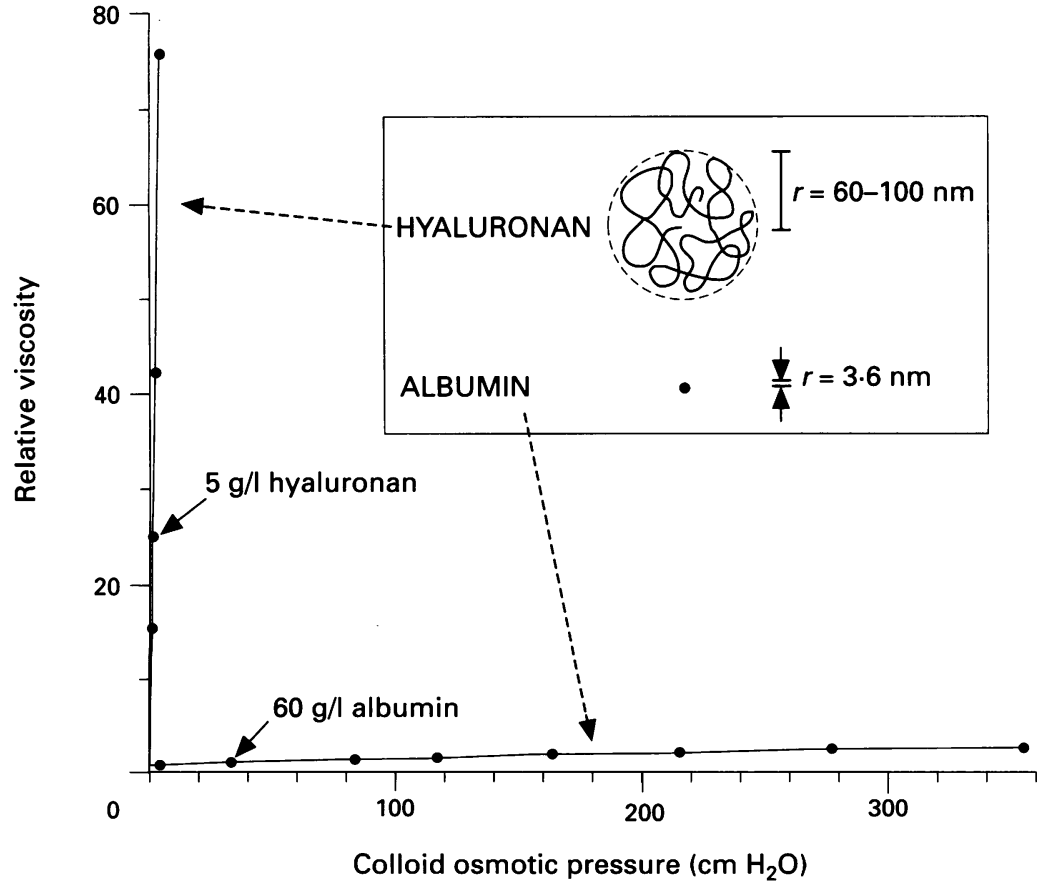

Figure 5 Effects of hyaluronan and albumin on colloid osmotic pressure (at $35^{\circ} \mathrm{C}$ and pH 7.4) and viscosity (at $35^{\circ} \mathrm{C}$ and wall shear stress of 29 dyn $\mathrm{cm}^{-2}$ ). Points represent increasing concentrations. Molecular dimension are compared in the inset, roughly to scale. $r=$ Molecular radius. showed that the anomalous viscosity of a suspension of particles (radius $r$ ) is given by:

$$
1 / \eta_{\text {apparent }}=1-\left\{\left(R_{\text {core }} / R_{\text {tube }}\right)^{4}\left(1-\left(1 / \eta_{\text {core }}\right)\right)\right\}
$$

where $\eta_{\text {apparent }}$ and $\eta_{\text {core }}$ are the apparent relative viscosity and the bulk viscosity of the liquid in the non-excluded core, respectively, solvent relative viscosity is 1 , and the radius of the nonexcluded region, $R_{\text {core, }}$ is $R_{\text {tube }}-0.735 r$. In extrapolating this to a biopolymer matrix, the equivalent tube radius of the irregular channels between the molecular fibres is calculated as the tube radius that produces the same fractional steric exclusion of particle (albumin molecule here) as the matrix. The resulting theoretical curves deviate above the Darcy line (line of equality, figure 1), but less so than the observed results. Possible reasons for the discrepancy between experiment and theory have been assessed previously. ${ }^{8}$

\section{Effect of intra-articular hyaluronan on trans-synovial flow $\dot{Q}_{\text {s }}$}

The physical properties of hyaluronan $(3-4 \mathrm{~g} / \mathrm{l}$; mol.wt $>10^{6}$ ) contrast strongly with those of albumin (fig 5). The COP of hyaluronan is slight, but its effect on viscosity is great. The effect of hyaluronan on $\dot{Q}_{\text {s }}$ is modest at low IAP, but remarkable at high IAP (fig 6). In the experiment illustrated, a fixed concentration of hyaluronan ( 3 or $6 \mathrm{~g} / \mathrm{l}$ ) was infused and IAP was increased every 15 to 20 minutes to determine the IAP- $\dot{Q}_{s}$ relation. The control curve with Krebs solution shows the well known increase in slope with IAP, indicating a decrease in hydraulic resistance with increasing IAP (Edlund curve ${ }^{11}$ ). This is attributable partly to stretching of the lining with pressure ${ }^{29}$ and partly to dilution of interstitial matrix biopolymers. ${ }^{23}$ Albumin reduces absorption rate in vivo at any given pressure (see above),

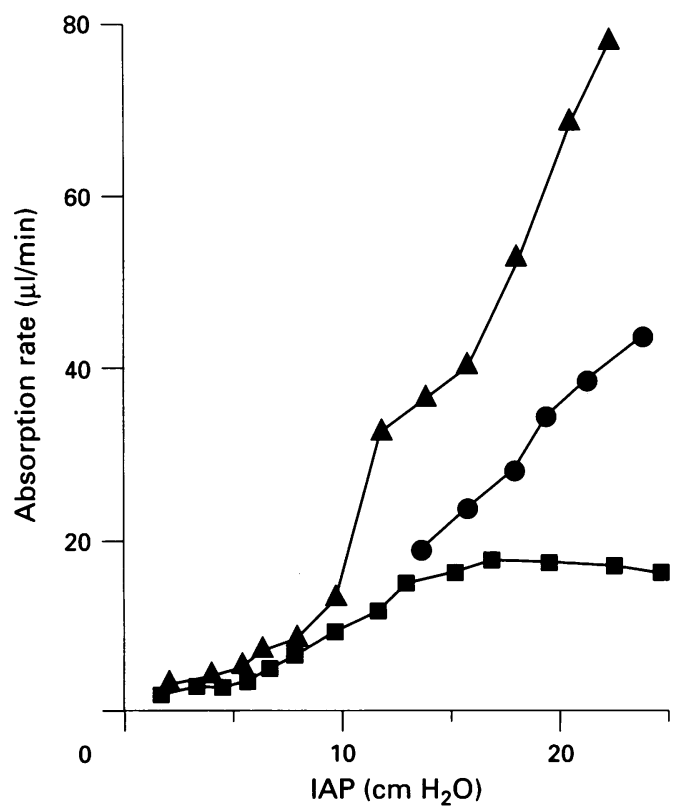

Figure 6 Effect of intra-articular pressure on net transsynovial flow out of joint cavity (absorption) for Krebs solution ( $\mathbf{\Delta})$, albumin $110 \mathrm{~g} /$ solution ( ) and hyaluronan $6 \mathrm{~g} /$ solution (1) in three individual rabbit knees. 


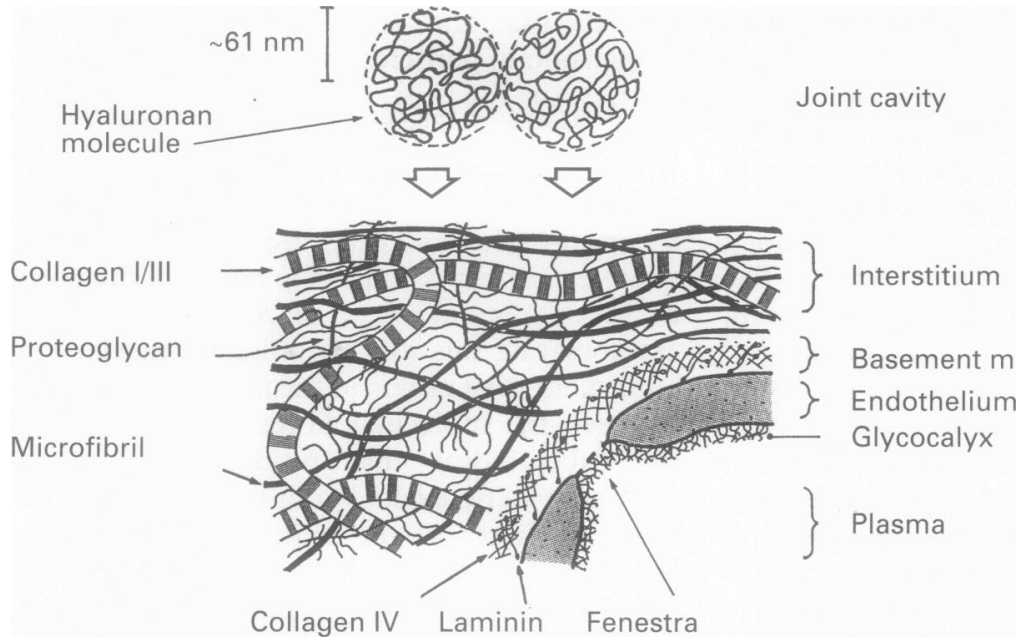

Figure 7 Sketch showing composition of pathway between joint cavity and plasma, comprising fibrous matrices of synovial interstitium, capillary basement membrane ( $m$.) and endothelial glycocalyx. The voluminous hyaluronan molecules of synovial fluid are thought to permeate the lining less freely than water, leading to an accumulation of hyaluronan at the interface when the intra-articular pressure is increased.

but it does not alter the basic shape of the pressure-flow relation (middle curve, fig 6). Hyaluronan, by contrast, alters the basic shape of the pressure-flow relation. At low IAPs, flows are reduced by hyaluronan to between

A
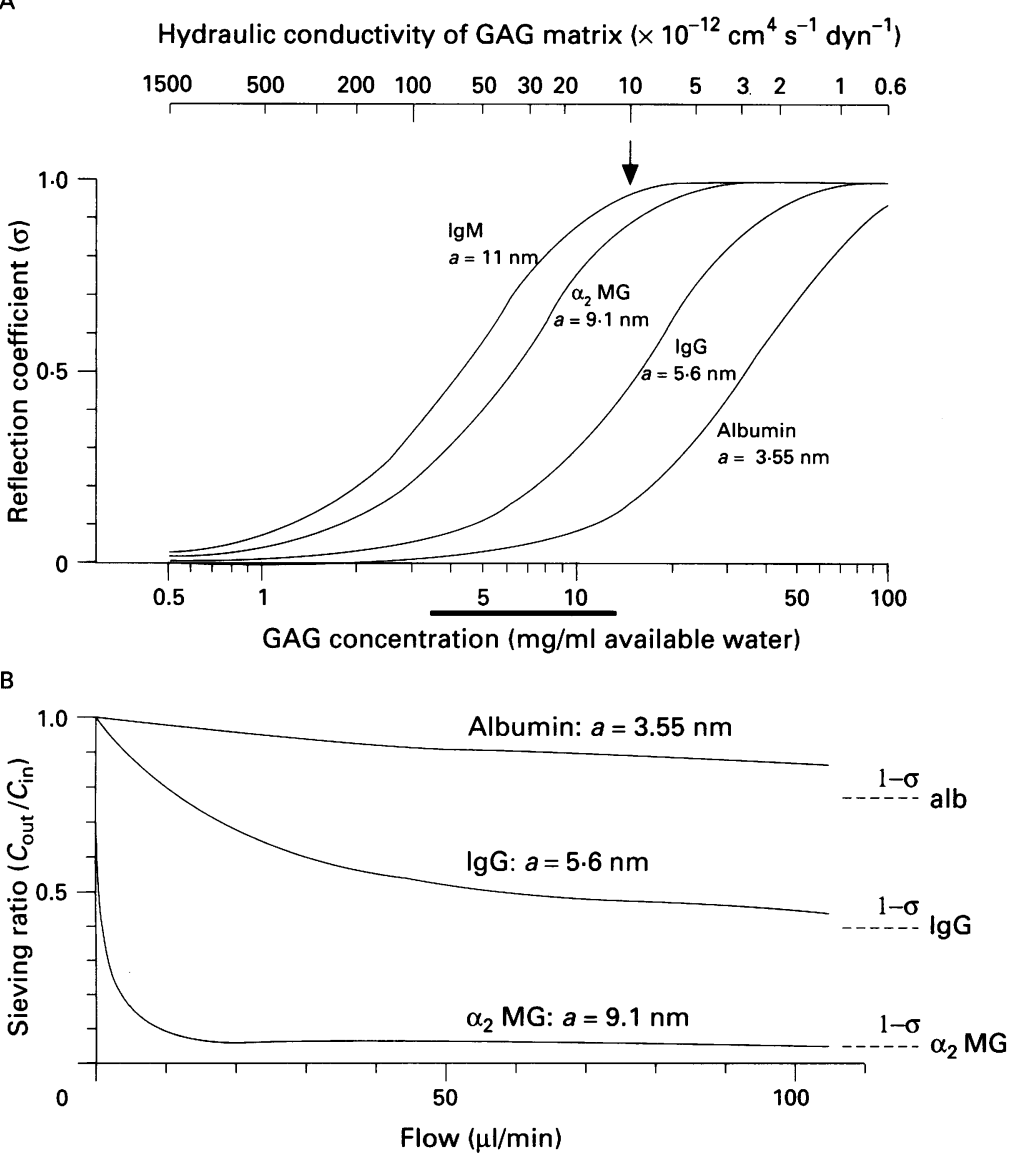

Figure 8 A: Relation between reflection coefficient $(\sigma)$ to a spherical macromolecule of radius $a$ and the concentration of glycosaminoglycan $(G A G)$ per ml of extrafibrillar space, modelled as random rigid cylindrical rods of radius $0.6 \mathrm{~nm}$ (see table). Dark bar shows currently estimated range for biopolymer concentration in rabbit knee synovium (see text). Small arrow shows estimated conductivity to saline at $35^{\circ} \mathrm{C} . \quad$ B: Theoretical dependence of sieving ratio (outflow concentration/inflow concentration, e.g. lymph concentration/intra-articular concentration) on flow, for a matrix with a flow-independent reflection coefficient. The ratio is close to 1 for all molecules at very slow flows. Adapted from Levick. ${ }^{33} \alpha_{2} M G=\alpha_{2}$-Macroglobulin.
$33 \%$ and $66 \%$ of control flow-a surprisingly modest effect considering that hyaluronan reduces the bulk fluidity to $6-10 \%$ of control fluidity. ${ }^{30}$ At greater IAPs, trans-synovial flow decreases increasingly below the control curve and begins to plateau. In several joints, including that illustrated, further increases in IAP then result, remarkably, in a slight decrease in the steady state $\dot{Q}_{\mathrm{s}}$. A reduction in steady state flow upon increasing IAP indicates an increasing resistance to flow. If subsynovial pressure does not change substantially and is close to atmospheric pressure (there are grounds for such an assumption), the IAP required to drive unit flow across the synovial lining is an index of synovial lining hydraulic resistance, and this is found to increase progressively as a function of IAP. With Krebs solution, by contrast, resistance decreases with increasing IAP, as has been known since the work of Edlund. ${ }^{11}$

\section{Hypothesis: hyaluronan molecular filtercake at the synovial surface?}

A phenomenon analogous to that shown in figure 6 occurs in vitro; ${ }^{31}$ when a hyaluronan solution is driven through a Millipore membrane (pore width $0.45 \mu \mathrm{m}$ ), the resistance to flow increases, because a filtercake of hyaluronan (or 'concentration polarisation layer') forms at the surface. The hydrated, mutually overlapping molecular domains of the vast hyaluronan molecules are too large to pass easily through the pores, so they accumulate just upstream and create a high resistance to the passage of water. ${ }^{32}$ As the equivalent tube radius within synovial interstitium is much less than $0.45 \mu \mathrm{m},{ }^{822}$ hyaluronan molecules may filtercake at the synovial surface also (fig 7). A preliminary study using ruthenium red to stain polyanionic material supports this idea: electron micrographs showed an accumulation of ruthenium red positive material just within the superficial interstitium and at the surface after infusion of hyaluronan solution under pressure. ${ }^{30}$

Hyaluronan can thus affect the hydraulic resistance of the joint cavity lining, and by acting as a 'dynamic waterproofing' during periods of joint flexion and increased IAP it may help the synovial lining retain the vital synovial fluid. A great deal more work remains to be done on this phenomenon, such as exploring the importance of hyaluronan concentration and chain length, which are subnormal in rheumatoid arthritis.

\section{Is synovium freely and equally permeable to all macromolecules?}

There is controversy as to whether the synovial lining matrix is sufficiently dense to impede the egress of IA macromolecules selectively (on the basis of size) as implied above. The limited evidence has been reviewed previously. ${ }^{33}$ Briefly, size selectivity has not been detected in the human knee upon comparing large and small globular plasma proteins, but there is some evidence for size based selectivity in the 
non-inflamed rabbit knee comparing albumin with proteoglycan or, as here, albumin with hyaluronan. Size selectivity is expected in a polymer matrix on the theoretical grounds that steric exclusion is closely linked to the generation of reflection coefficients (table). Figure 8A shows the theoretical relation between the synovial reflection coefficient to globular macromolecules and biopolymer concentration (modelled as randomly distributed cylinders of radius $0.6 \mathrm{~nm}$ ) or hydraulic permeability (related to concentration ${ }^{22}$ (table)). In the region of interest for synovium (dark bar and arrow in figure 8A), the reflection coefficients of the larger plasma proteins are unlikely to be zero and may exceed $0 \cdot 1$. While the maximum molecular separation is $1-\sigma$ (i.e. the smallest effluent:input ratio is $1-\sigma$, for example $<0.9$ for $\sigma>0.1$ ), the actual separation depends equally on trans-synovial fluid velocities: as filtration velocity is reduced, diffusion increasingly offsets molecular sieving and increases the ratio towards unity (fig 8B).

The view that synovium is less permeable to hyaluronan than to albumin implies macromolecular selectivity. In support of this, the bulk turnover of IA water and protein is estimated to be an order of magnitude faster than the turnover of IA hyaluronan. The turnover time for synovial fluid volume and protein is estimated to be about one hour in rabbit and normal human knees, ${ }^{5}$ while that for hyaluronan in the rabbit shoulder is $20-28$ hours. ${ }^{34}$ Similarly, in the rabbit knee hyaluronan has a half life of 27-32 hours at normal volume, ${ }^{35}$ or between 13 hours (mol.wt $6 \times 10^{6}$ ) and 10 hours (mol.wt $0.9 \times 10^{6}$ ) in volume expanded knees. ${ }^{36}$ Large differences between hyaluronan and water/albumin turnover times seem to imply selective retention of hyaluronan within the joint cavity and therefore a selective capacity for the lining tissue.

This work was supported by grants from the Arthritis and Rheumatism Council and the Wellcome Trust (031333/Z/90/A 039033/Z/93/Z/1·27).

1 Stevens C R, Blake D R, Merry P, Revell P A, Levick J R A comparative study by morphometry of the microvasculature in normal and rheumatoid synovium. Arthritis Rheum 1991; 34: 1508-13.

2 Levick J R, Smaje L H. An analysis of the permeability of a fenestra. Microvasc Res 1987; 33: 233-56.

3 Knight A D, Levick J R, McDonald J N. Relation between trans-synovial flow and plasma colloid osmotic pressure, with an estimation of the albumin reflection coefficient in the rabbit knee. $Q \mathcal{F}$ Exp Physiol 1988; 73: 47-66.

4 Levick J R. Blood flow and mass transport in synovial joints. In: Renkin E M, Michel C C, eds. Handbook of Physiology. In: Renkin $\mathrm{M}$, Michel C C, eds. Handbook of Physiology. Section 2, The Cardiovascular System, Volume IV, The
Microcirculation, ch 19, Bethesda: American Physiology Microcirculation, ch 19,

5 Levick J R. Synovial fluid and trans-synovial flow in stationary and moving joints. In: Helminen $\mathrm{H}$, Kiviranta I, Tammi M, Saamaren A M, Paukonnen K, Jurvelin J, eds. foint Loading: Biology and Health of Articular Structures. Bristol: Wright \& Sons, 1987; 149-86.

6 Henderson B, Edwards J C W. The synovial lining. London Chapman \& Hall, 1987.
7 Levick J R. A two-dimensional morphometry-based model of interstitial and transcapillary flow in rabbit synovium. Exp Physiol 1991; 76: 905-21.

8 Levick J R. An analysis of the interaction between extravascular plasma protein, interstitial flow and capillary viltration; application to synovium. Microvasc Res 1994; 47: $90-125$.

9 Simkin P A, Benedict R S. Iodide and albumin kinetics in normal canine wrists and knees. Arthritis Rheum 1990; 33 73-9.

10 Jensen L T, Henriksen J H, Olesen H P, Risteli J, Lorenzen I. Lymphatic clearance of synovial fluid in conscious pigs; the aminoterminal propeptide of type III procollagen. Eur f Clin Invest 1993; 23: 778-84.

11 Edlund T. Studies on the absorption of colloids and fluid from rabbit knee joints. Acta Physiol Scand 1949; 18 (suppl 62): 1-108.

12 Levick J R, Knight A D. Interaction of plasma colloid osmotic pressure and joint fluid pressure across the endothelium-synovium layer; significance of extravascular resistance. Microvasc Res 1988; 35: 109-21.

13 Patlack C S, Goldstein D A, Hoffman J F. The flow of solute and solvent across a two-membrane system. $\mathcal{F}$ Theor Biol 1963; 5: 426-42.

14 Comper W D, Zamparo O. Hydraulic conductivity of polymer matrices. Biophys Chem 1989; 34: 127-35.

15 Ogston A G. On the interaction of solute molecules with porous networks. F Physical Chem 1970; 74: 668-9.

16 Curry F E. Mechanics and thermodynamics of transcapillary exchange. In: Renkin E M, Michel C C, System, Volume IV, The Microcirculation, ch 8. Bethesda: American Physiology Society, 1984; 309-74.

17 Anderson J L, Malone D M. Mechanism of osmotic flow in porous membranes. Biophys $\mathcal{F} 1974 ; 14: 957-82$.

18 Whitmore R L. Rheology of the circulation. Oxford: Pergamon Press, 1968.

19 Kedem O, Katchalsky A. Thermodynamic analysis of the permeability of biological membranes to non-electrolytes. Biochim Biophys Acta 1958; 27: 229-45.

20 Ogston A G, Preston B N, Wells J D. On the transport of compact particles through solutions of chain polymers. Proc $R$ Soc $A$ 1973; 333: 297-316.

21 Rippe B, Haraldsson B. Transport of macromolecules across microvascular walls: the two-pore theory. Physiol Rev 1994; 74: 163-219.

22 Levick J R. Flow through interstitium and other fibrous matrices. $Q \mathcal{F}$ Exp Physiol 1987; 72: 409-38.

23 Mason $R$ M, Price F M, Levick J R. A quantitative investigation of the glycosaminoglycans of the synovium. investigation of the glycosaminogly

24 Levick J R, McDonald J N. Viscous and osmotically mediated changes of interstitial flow induced by extravascular albumin in synovium. Microvasc Res 1994; 47: 68-89.

25 Ogston A G. The spaces in a uniformly random suspension of fibres. Trans Faraday Soc 1958; 54: 1754-7.

26 Aukland K, Reed R K. Interstitial-lymphatic mechanisms in the control of extracellular fluid volume. Physiol Rev 1993; 73: 1-78.

27 Goldsmith H L, Cokelet G R, Gaehtgens P. Robin Fahreus; evolution of his concepts in cardiovascular physiology. Am 7 Physiol 1989; 257: H1005-15.

28 Happel J, Brenner H. Low Reynolds number hydrodynamics. Englewood Cliffs: Prentice-Hall, 1965; 392-404.

29 Levick J R, McDonald J N. Ultrastructure of transport pathways in stressed synovium of the knee in pathways in stressed synovium of the knee
anaesthetized rabbits. $\mathcal{A}$ Physiol $1989 ; 419: 493-508$.

$30 \mathrm{McDonald}$ J N, Levick J R. Hyaluronan reduces fluid escape rate from joints disparately from its effect on fluidity. Exp Physiol 1994; 79: 103-6.

31 Parker K H, Winlove C P. The macromolecular basis of the hydraulic conductivity of the arterial wall. Biorheology 1984; 21: 181-96.

32 Johnson M, Kamm R, Ethier C R, Pedley T. Scaling laws and the effect of concentration polarization on the permeability of hyaluronic acid. Physicochemical Hydrodynamics 1987;9: 427-41.

33 Levick J R. Synovial fluid; determinants of volume and material concentration. In: Kuettner $\mathrm{K}$ E, Hascall V C, material concentration. In: Kuettner K E, Hascall V C, New York: Raven Press, 1992; 529-41.

34 Knox P, Levick J R, McDonald J N. Synovial fluid-its mass, macromolecular content and pressure in major limb joints of the rabbit. $Q \mathcal{F}$ Exp Physiol 1988; 73: 33-46.

35 Denlinger J L. Metabolism of sodium hyaluronate in articular and ocular tissues [dissertation]. Lille: Université de Sciences et Techniques de Lille, 1982 .

36 Brown T J, Laurent U B G, Fraser J R E. Turnover of hyaluronan in synovial joints. Exp Physiol 1991; 76: 125-34. 